\title{
Oscillation Theorems for Non-Canonical Self-Adjoint Differential Equations of Second Order
}

\author{
J. Ohriska
}

Abstract. The report deals with the equation $\left(r(t) u^{\prime}(t)\right)^{\prime}+p(t) u(t)=0$ and renders effective sufficient conditions for its oscillation and non-oscillation in the case $\int^{\infty} \frac{d t}{r(t)}<\infty$.

Keywords: Oscillation

AMS subject classification: $34 \mathrm{C} 10$

For $t_{0} \geq 0$ we consider the second order self-adjoint differential equation

$$
\left(r(t) u^{\prime}(t)\right)^{\prime}+p(t) u(t)=0 \quad\left(t \geq t_{0}\right)
$$

Throughout the paper we shall assume that

(i) $r \in C\left[t_{0}, \infty\right), r(t)>0$ for all $t \geq t_{0}$ and $\int^{\infty} \frac{d t}{r(t)}<\infty$

(ii) $p \in C\left[t_{0}, \infty\right)$.

We say that equation (1) is in canonical form if $\int^{\infty} \frac{d t}{r(t)}=\infty$, and that it is in non-canonical form if $\int^{\infty} \frac{d t}{r(t)}<\infty$. By a solution of equation (1) we mean a function $u:\left[t_{0}, \infty\right) \rightarrow \mathbb{R}$ with the properties $u \in C^{1}\left[t_{0}, \infty\right)$ and $r u^{\prime} \in C^{1}\left[t_{0}, \infty\right)$ which satisfies equation (1) for all $t \in\left[t_{0}, \infty\right)$ and is not identically zero. Such a solution is called oscillatory if it has arbitrarily large zeros, and non-oscillatory otherwise. An equation is said to be oscillatory if one, and thereby each solution is oscillatory, otherwise it is said to be nonoscillatory.

J. Ohriska: P. J. Safarik University, Dept. Math., Jesenna 5, 04154 Kosice, Slovak Rep.; ohriska@kosice.upjs.sk. This research was supported by grant 1/7466/20 of Slovak Grant Agency.

ISSN 0232-2064 / \$2.50 C Heldermann Verlag Berlin 
Equation (1) and its special case

$$
u^{\prime \prime}(t)+p(t) u(t)=0
$$

is still a valid subject for research. Although there are many results concerning the oscillatory nature of equations (1) or (2), no completely satisfactory answer has yet been obtained. In oscillation theory, one of the tools for obtaining new results is a transformation of an equation considered. We recall at least the well known Riccati, Kummer and Bohl transformations (see, e.g., [9]). On the other hand, in articles which deal with equation (1) the authors very often require $r \in C^{1}\left[t_{0}, \infty\right)$ and define a solution as a function $u \in C^{2}\left[t_{0}, \infty\right)$ (see, e.g., $[3,4,8,9])$.

Note that in the case when equation (1) is in the canonical form, there is one significant analogy with equation (2), namely that the integrals $\int^{\infty} \frac{d t}{r(t)}$ and $\int^{\infty} 1 d t$ are divergent. This fact gives the possibility to make some steps in the study of equation (1) in analogous to the study of equation (2). In the non-canonical case this is not posssible and the study of such equation is then more difficult.

Since equation (2), as special case of (1), is measured more than equation (1), our purpose in this article is to extend some results known for equation (2) to equation (1) by using a useful transformation. For this we put

$$
\rho(t)=\int_{t}^{\infty} \frac{d s}{r(s)} \quad\left(t \geq t_{0}\right)
$$

and we mention the following result which is a special case of [6: Theorem $2.1]$.

Theorem A. Let the conditions

(a) $I$ and $I_{1}$ are intervals in $\mathbb{R}$

(b) $v \in C\left(I_{1}\right), v: I_{1} \rightarrow I$ strictly monotone

(c) $p \in C(I), p: I \rightarrow \mathbb{R}$

be satisfied. A function $u(t)$ is a solution of the equation

$$
y^{\prime \prime}(t)+p(t) y(t)=0 \quad(t \in I)
$$

if and only if the function $u(v(t))$ is a solution of the equation

$$
\frac{d^{2} y(s)}{d v^{2}}+p(v(s)) y(s)=0 \quad\left(s \in I_{1}\right)
$$

Note that

$$
\frac{d f(t)}{d v}=\lim _{x \rightarrow t} \frac{f(x)-f(t)}{v(x)-v(t)}
$$


we call the $v$-derivative of the function $f$ at the point $t$. Then the expression $\frac{d^{2} y(s)}{d v^{2}}$ is the second $v$-derivative of the function $y$ at the point $s$ and

$$
\frac{d^{2} y(s)}{d v^{2}}=\frac{d}{d v}\left(\frac{d y(s)}{d v}\right)=\frac{1}{v^{\prime}(s)}\left(\frac{y^{\prime}(s)}{v^{\prime}(s)}\right)^{\prime}
$$

if there exist $v^{\prime}(s) \neq 0$ and $y^{\prime}(s)$ for $s \in I_{1}$. The process of obtaining equation (4) from (3) we call the $v$-transformation of equation (3). It is also useful to note that if $\phi$ is the inverse function to $v$, so the $\phi$-transformation of (4) gives again equation (3). For more detailed information about the $v$-derivative and the $v$-transformation we refer the reader to [6].

Now we are ready to introduce the following result.

Theorem 1. Let assumptions (i) and (ii) be satisfied. Then equation (1) is oscillatory if

$$
\liminf _{t \rightarrow \infty} \rho^{2}(t) r(t) p(t)>\frac{1}{4},
$$

and it is non-oscillatory provided

$$
\limsup _{t \rightarrow \infty} \rho^{2}(t) r(t) p(t)<\frac{1}{4} .
$$

Proof. From the definition of the function $\rho$ we see that $\rho \in C^{1}\left[t_{0}, \infty\right)$ is decreasing and $\rho:\left[t_{0}, \infty\right) \rightarrow\left(0, \rho_{0}\right]$ with $\rho_{0}=\rho\left(t_{0}\right)$. Denote by $\phi$ the inverse function to $\rho$. Now using the notion of the $v$-derivative of a function (see [5] or [6]) we can write equation (1) in the form

$$
\frac{d^{2} u(t)}{d \rho^{2}}+r(t) p(t) u(t)=0 \quad\left(t \geq t_{0}\right)
$$

The $v$-transformation (see [5] or [6]) of this equation, with $v=\phi$, yields the equation

$$
y^{\prime \prime}(s)+r(\phi(s)) p(\phi(s)) y(s)=0 \quad\left(0<s \leq \rho_{0}\right)
$$

and we know that a function $y=y(s)\left(s \in\left(0, \rho_{0}\right]\right)$ is a solution of equation (7) if and only if $u(t)=y(\rho(t))\left(t \in\left[t_{0}, \infty\right)\right)$ is a solution of equation (1). Now we transform equation (7) by change of the independent variable. Putting $x=\frac{1}{s}$ and $z(x)=y\left(\frac{1}{x}\right)$ we obtain the equation

$$
\left(x^{2} z^{\prime}(x)\right)^{\prime}+\frac{1}{x^{2}} r\left(\phi\left(\frac{1}{x}\right)\right) p\left(\phi\left(\frac{1}{x}\right)\right) z(x)=0 \quad\left(x \in\left[\frac{1}{\rho_{0}}, \infty\right)\right) .
$$

From the above transformations we see that either equations (1) and (8) are oscillatory or both are non-oscillatory. Now, for $a \in \mathbb{R}$, we take into consideration the Euler equation

$$
\left(x^{2} w^{\prime}(x)\right)^{\prime}+a w(x)=0
$$


which is oscillatory if $a>\frac{1}{4}$ and non-oscillatory if $a \leq \frac{1}{4}$. Using the Sturm comparison theorem to equations (8) and (9) we know that equation (8) (and thus also equation (1)) is oscillatory if

$$
\liminf _{x \rightarrow \infty} \frac{1}{x^{2}} r\left(\phi\left(\frac{1}{x}\right)\right) p\left(\phi\left(\frac{1}{x}\right)\right)>\frac{1}{4} .
$$

But using the substitution $x=\frac{1}{\rho(t)}$ we get

$$
\liminf _{x \rightarrow \infty} \frac{1}{x^{2}} r\left(\phi\left(\frac{1}{x}\right)\right) p\left(\phi\left(\frac{1}{x}\right)\right)=\liminf _{t \rightarrow \infty} \rho^{2}(t) r(t) p(t)
$$

and the first part of Theorem 1 is proved. The second part can be proved in a similar way

Note that Theorem 1 is an analogy of [5: Theorem 2.3] concerning differential equation (1) in canonical form when the role of the function $\rho^{2}(t) r(t) p(t)$ appearing in conditions (5) and (6) takes over the function $R^{2}(t) r(t) p(t)$ where $R(t)=\int_{t_{0}}^{t} \frac{d s}{r(s)}$. Hence the result due to E. Hille (see [7: p. 194]) which says that equation (2) is oscillatory if $\liminf _{t \rightarrow \infty} t^{2} p(t)>\frac{1}{4}$ and non-oscillatory if $\lim \sup _{t \rightarrow \infty} t^{2} p(t)<\frac{1}{4}$ we have transferred to equation (1) in both the canonical and the non-canonical cases.

In the following example we remit on a relation of Theorem 1 to another result known for equation (1).

Example 1. We consider the non-canonical differential equation

$$
\left((t+1)^{2} u^{\prime}(t)\right)^{\prime}+(t+1)^{2} u(t)=0 \quad(t \geq 0) .
$$

It is easy to see that

$$
\begin{aligned}
I_{r} & =\int_{0}^{\infty} \frac{d t}{r(t)}=1<\infty \\
I_{p r} & =\int_{0}^{\infty} p(t) \int_{0}^{t} \frac{d s}{r(s)} d t=\infty \\
I_{r p} & =\int_{0}^{\infty} \frac{1}{r(t)} \int_{0}^{t} p(s) d s d t=\infty
\end{aligned}
$$

in the case of equation $(10)$ and thus by $\left[1\right.$ : Theorem $\left.1 / i_{4}\right]$ we know that every solution $u$ of equation (10) is either oscillatory or such that $u(t) u^{\prime}(t)<0$ for sufficiently large $t$ and $\lim _{t \rightarrow \infty} u(t)=0$. On the other hand, $\lim _{t \rightarrow \infty} \rho^{2}(t) r(t) p(t)=$ $\infty$ and by our Theorem 1 it is clear that equation (10) is oscillatory what is a stronger assertion than the previous one. Note that one solution of equation (10) is the function $u(t)=\frac{\sin (t+1)}{t+1}$. 
We see that the above Theorem 1 can not be used if $\rho^{2}(t) r(t) p(t) \rightarrow \frac{1}{4}$ as $t \rightarrow \infty$. The same problem in the case of equation (2) was solved by P. Hartman [2], and thus we know that equation (2) is oscillatory or nonoscillatory if

$$
\liminf _{t \rightarrow \infty} t^{2} \ln ^{2} t\left[p(t)-\frac{1}{4 t^{2}}\right]>\frac{1}{4} \quad \text { or } \quad \quad \limsup _{t \rightarrow \infty} t^{2} \ln ^{2} t\left[p(t)-\frac{1}{4 t^{2}}\right]<\frac{1}{4},
$$

respectively. In the case of equation (1) we have the following analogy of Hartman's result.

Theorem 2. Let assumptions (i) and (ii) be satisfied. Then equation (1) is oscillatory if

$$
\liminf _{t \rightarrow \infty} \ln ^{2}\left(\ln \left(\frac{1}{\rho(t)}\right)\right)\left[4 \rho^{2}(t) r(t) p(t) \ln ^{2}\left(\frac{1}{\rho(t)}\right)-\ln ^{2}\left(\frac{1}{\rho(t)}\right)-1\right]>1
$$

and non-oscillatory if

$$
\limsup _{t \rightarrow \infty} \ln ^{2}\left(\ln \left(\frac{1}{\rho(t)}\right)\right)\left[4 \rho^{2}(t) r(t) p(t) \ln ^{2}\left(\frac{1}{\rho(t)}\right)-\ln ^{2}\left(\frac{1}{\rho(t)}\right)-1\right]<1 .
$$

Proof. Consider equation (8) on some interval $\left[x_{0}, \infty\right)$ where $x_{0}>1$ and transform it by a change of the dependent and independent variables in the form

$$
x=e^{s} \quad \text { and } \quad z(x)=x^{-\frac{1}{2}} V(s) .
$$

Then equation (8) acquires the form

$$
V^{\prime \prime}(s)+\left(e^{-2 s} r\left(\phi\left(e^{-s}\right)\right) p\left(\phi\left(e^{-s}\right)\right)-\frac{1}{4}\right) V(s)=0 \quad\left(\ln x_{0} \leq s<\infty\right) .
$$

From (11) we see that equation (12) is oscillatory if and only if equation (8) is oscillatory. This means that equation (12) is oscillatory if and only if equation (1) is oscillatory. With regard to Hartman's result equation (12) is oscillatory if

$$
\liminf _{s \rightarrow \infty} s^{2} \ln ^{2} s\left[e^{-2 s} r\left(\phi\left(e^{-s}\right)\right) p\left(\phi\left(e^{-s}\right)\right)-\frac{1}{4}-\frac{1}{4 s^{2}}\right]>\frac{1}{4} .
$$

But using the substitution $s=\ln \frac{1}{\rho(t)}$ we get

$$
\begin{aligned}
& \liminf _{s \rightarrow \infty} s^{2} \ln ^{2} s\left[e^{-2 s} r\left(\phi\left(e^{-s}\right)\right) p\left(\phi\left(e^{-s}\right)\right)-\frac{1}{4}-\frac{1}{4 s^{2}}\right] \\
& \quad=\frac{1}{4} \liminf _{t \rightarrow \infty} \ln ^{2}\left(\ln \left(\frac{1}{\rho(t)}\right)\right)\left[4 \rho^{2}(t) r(t) p(t) \ln ^{2}\left(\frac{1}{\rho(t)}\right)-\ln ^{2}\left(\frac{1}{\rho(t)}\right)-1\right]
\end{aligned}
$$

and the proof of the first part of Theorem 2 is complete. The second part can be proved in a similar way 
We illustrate the meaning of Theorem 2 by the following example.

Example 2. We consider the differential equation

$$
\left(t^{3} u^{\prime}(t)\right)^{\prime}+t u(t)=0
$$

In this case $\lim _{t \rightarrow \infty} \rho^{2}(t) r(t) p(t)=\frac{1}{4}$ and thus Theorem 1 is not applicable. However,

$$
\limsup _{t \rightarrow \infty} \ln ^{2}\left(\ln \left(\frac{1}{\rho(t)}\right)\right)\left[4 \rho^{2}(t) r(t) p(t) \ln ^{2}\left(\frac{1}{\rho(t)}\right)-\ln ^{2}\left(\frac{1}{\rho(t)}\right)-1\right]=-\infty
$$

and by Theorem 2 we know that equation (13) is non-oscillatory. Note that one solution of equation (13) is the function $u(t)=\frac{1}{t}$.

\section{References}

[1] Cecchi, M., Marini, M. and G. Villari: Integral criteria for a classification of solutions of linear differential equations. J. Diff. Equ. 99 (1992), 381 - 397.

[2] Hartman, P.: Ordinary Differential Equations. New York et al.: John Wiley \& Sons 1964.

[3] Li, H. J.: Oscillation criteria for second order linear differential equations. J. Math. Anal. Appl. 194 (1995), 217 - 234.

[4] Li, H. J. and Ch. Ch. Yeh: Oscillation and nonoscillation criteria for second order linear differential equations. Math. Nachr. 194 (1998), 171 - 184.

[5] Ohriska, J.: On the oscillation of a linear differential equation of second order. Czechoslovak Math. J. 39 (114) (1989), 16 - 23.

[6] Ohriska, J.: Oscillation of differential equations and v-derivatives. Czechoslovak Math. J. 39 (114) (1989), $24-44$.

[7] Reid, W. T.: Sturmian Theory for Ordinary Differential Equations. New York et al.: Springer-Verlag 1980.

[8] Rogovchenko, Y. V.: Note on "Oscillation criteria for second order linear differential equations". J. Math. Anal. Appl. 203 (1996), 560 - 563.

[9] Willett, D.: Classification of second order linear differential equations with respect to oscillation. Adv. Math. 3 (1969), $594-623$.

Received 18.05.2001; in revised form 12.02.2002 